\title{
Corrigendum: Safety signal dampens reception for mipomersen antisense
}

Jim Kling

Nat. Biotechnol. 28, 295-297 (2010); published online 8 April 2010; corrected after print 9 July 2010

In the version of this article initially published, some of the oligos in Table 1 are described as phosphorothioate modified. In fact, all antisense oligonucleotides are phosphorothioate-modified oligos. In addition, Lucanix, which is not an antisense oligo, has been removed from the table. The error has been corrected in the HTML and PDF versions of the article.

\section{Corrigendum: $A b$ initio reconstruction of cell type-specific transcriptomes in mouse reveals the conserved multi-exonic structure of lincRNAs}

Mitchell Guttman, Manuel Garber, Joshua Z Levin, Julie Donaghey, James Robinson, Xian Adiconis, Lin Fan, Magdalena J Koziol, Andreas Gnirke, Chad Nusbaum, John L Rinn, Eric S Lander \& Aviv Regev

Nat. Biotechnol. 28, 503-510 (2010); published online 02 May 2010; corrected after print 9 July 2010

In the version of this article initially published, the fourth sentence in the Online Methods section "RNA extraction and library preparation," that read in part "procedure that combines a random priming step with a shearing step $8,9,28$ and results in fragments of $\sim 700$ bp in size," should have read, "procedure that combines fragmentation of mRNA to a peak size of $\sim 750$ nucleotides by heating ${ }^{6}$ followed by random-primed reverse transcription ${ }^{8}$." The error has been corrected in the HTML and PDF versions of the article.

\section{Erratum: US biodefense contracts continue to lure biotechs}

\section{Catherine Shaffer}

Nat. Biotechnol. 28, 187-188 (2010); published online 8 March 2010; corrected after print DD Month 9 July 2010

In the version of this article initially published, in Table 1, the Emergent BioSolutions' anthrax countermeasures in development listed AV-7909 as being in phase 2 under a $\$ 447.6$ million BARDA contract; AV-7909 is in phase 1 and the BARDA contract is for $\$ 29.7$ million. AIGIV is in phase $1 / 3$, not phase $1 / 2$. Finally, a third product was omitted; anthrax monoclonal is in preclinical testing under a $\$ 24$ million BARDA contract. The \$447.6 million BARDA contract was for procurement and product enhancements on BioThrax. Also, on p.188, column 2, line 7, the vaccine requires five injections, not six as originally stated. The error has been corrected in the HTML and PDF versions of the article.

\section{Erratum: Single base-resolution methylome of the silkworm reveals a sparse epigenomic map}

Hui Xiang, Jingde Zhu, Quan Chen, Fangyin Dai, Xin Li, Muwang Li, Hongyu Zhang, Guojie Zhang, Dong Li, Yang Dong, Li Zhao, Ying Lin, Daojun Cheng, Jian Yu, Jinfeng Sun, Xiaoyu Zhou, Kelong Ma, Yinghua He, Yangxing Zhao, Shicheng Guo, Mingzhi Ye, Guangwu Guo, Yingrui Li, Ruiqiang Li, Xiuqing Zhang, Lijia Ma, Karsten Kristiansen, Qiuhong Guo, Jianhao Jiang, Stephan Beck, Qingyou Xia, Wen Wang \& Jun Wang

Nat. Biotechnol. 28, 516-520 (2010); published online 02 May 2010; corrected after print 9 July 2010

In the version of this article initially published, references 4 and 7 were inadvertently interchanged. The error has been corrected in the HTML and PDF versions of the article.

\section{Erratum: Up for grabs}

Michael Eisenstein

Nat. Biotechnol. 28, 544-546 (2010); published online 7 June 2010; corrected after print 9 July 2010

In the version of the article originally published, it was stated that the Cohen-Boyer patents generated hundreds of billions of dollars in licensing revenue. It should have read hundreds of millions of dollars. The error has been corrected in the HMTL and PDF versions of the article. 